\title{
Crystal Structure of the Avian Astrovirus Capsid Spike
}

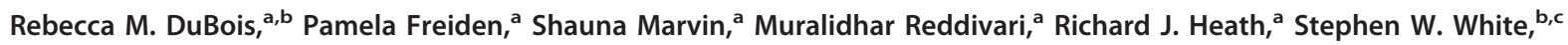 \\ Stacey Schultz-Cherry ${ }^{\mathrm{a}}$

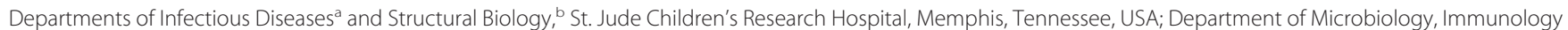 \\ and Biochemistry, University of Tennessee Health Science Center, Memphis, Tennessee, USAc
}

Astroviruses are small, nonenveloped, single-stranded RNA viruses that cause diarrhea in a wide variety of mammals and birds. On the surface of the viral capsid are globular spikes that are thought to be involved in attachment to host cells. To understand the basis of species specificity, we investigated the structure of an avian astrovirus capsid spike and compared it to a previously reported human astrovirus capsid spike structure. Here we report the crystal structure of the turkey astrovirus 2 (TAstV-2) capsid surface spike domain, determined to $1.5-\AA \AA$ resolution, and identify three conserved patches on the surface of the spike that are candidate avian receptor-binding sites. Surprisingly, the overall TAstV-2 capsid spike structure is unique, with only distant structural similarities to the human astrovirus capsid spike and other viral capsid spikes. There is an absence of conserved putative receptor-binding sites between the human and avian spikes. However, there is evidence for carbohydrate-binding sites in both human and avian spikes, and studies with human astrovirus 1 (HAstV-1) suggest a minor role in infection for chondroitin sulfate but not heparin. Overall, our structural and functional studies provide new insights into astrovirus host cell entry, species specificity, and evolution.

A stroviruses are small, nonenveloped RNA viruses that are associated with diarrhea in a wide variety of species. The Astroviridae family is composed of two genera, Mamastrovirus and Avastrovirus, that infect mammalian and avian species, respectively. Members of the Mamastrovirus genus include human astroviruses, which are one of the leading causes of gastroenteritis in children under the age of 2 years, immunocompromised people, and the elderly (1-3). Infection with human astrovirus serotype 1 (HAstV-1) is most commonly detected, although eight canonical serotypes of HAstV (HAstV-1 to -8) and several noncanonical human genogroups have been isolated with various frequencies $(4,5)$. In poultry, infections with members of the Avastrovirus genus have been shown to cause disease, growth defects, and mortality in a wide variety of poultry, including chickens, turkeys, and ducks (6-10).

Astroviruses possess single-stranded, positive-sense RNA genomes containing three open reading frames: ORF1a, ORF1b, and ORF2. These encode the viral nonstructural proteins, the viral RNA-dependent RNA polymerase, and the viral capsid protein, respectively (11-16). The astrovirus capsid is responsible for attachment and entry into host cells $(15,17)$. The capsid is a multidomain protein with a conserved $\mathrm{N}$-terminal region and a highly variable C-terminal region (18-22). The conserved N-terminal region, comprising the basic, $\mathrm{S}$, and $\mathrm{P} 1$ domains, encompasses the viral genome and forms the shell of the capsid $(20,23)$. The highly variable C-terminal region comprises the spike (also called the P2 domain) and acidic domains (23). The acidic domain is removed from the virion by host cell caspases $(24,25)$, leaving only the spikes projecting from the capsid shell surface. The capsid undergoes further maturation by host extracellular proteases to become an infectious particle. Recent cryo-electron microscopy studies on immature and mature astrovirus particles revealed that the capsid forms an icosahedral shell $(\mathrm{T}=3)$ studded with globular dimeric spikes (26).

The astrovirus capsid spike domain is thought to be important for host cell receptor binding, because anti-HAst $\mathrm{V}$ neutralizing monoclonal antibodies that map to the capsid spike domain are able to block virus attachment to host cells $(15,17)$. Although the astrovirus receptor is not known, the recently reported high-resolution crystal structure of the HAst $\mathrm{V}-8$ spike domain revealed a putative carbohydrate-binding pocket (23). It was further reported that heparin, heparan sulfate, or dextran sulfate was able to reduce HAstV-8 infectivity in Caco- 2 cells (23). The HAstV-8 spike domain structure also revealed distant structural similarity to the hepatitis E virus (HEV) spike domain, which is also hypothesized to have a carbohydrate-binding pocket (27-30), and heparan sulfate proteoglycans were found to be an essential component for cell attachment and HEV infection (31). Finally, the structurally related spike domain from norovirus has well-characterized receptor-binding sites for histo-blood group antigen carbohydrates $(32,33)$.

To understand the basis of species specificity, we investigated the structure of an avian astrovirus capsid spike and compared it to the previously reported HAstV-8 capsid spike structure. We sought to determine if the human and avian spikes, which lack sequence similarity, would have structural similarity and/or a conserved receptor-binding site. In addition, the avian structure is crucial for interpreting results from studies with the only astrovirus small-animal model: turkey astrovirus 2 (TAstV-2) infection in turkey poults (34). Here we used limited proteolysis to isolate a stable fragment of the TAstV-2 capsid corresponding to the surface-exposed spike domain, and we determined its crystal structure to $1.5-\AA$ resolution. We found that it has a unique structure, with only distant structural similarities to the human astrovirus capsid spike and other viral spikes. In contrast to the previous

\footnotetext{
Received 8 November 2012 Accepted 26 April 2013

Published ahead of print 8 May 2013

Address correspondence to Stacey Schultz-Cherry,

stacey.schultz-cherry@stjude.org

Copyright @ 2013, American Society for Microbiology. All Rights Reserved.

doi:10.1128/JVI.03139-12
} 

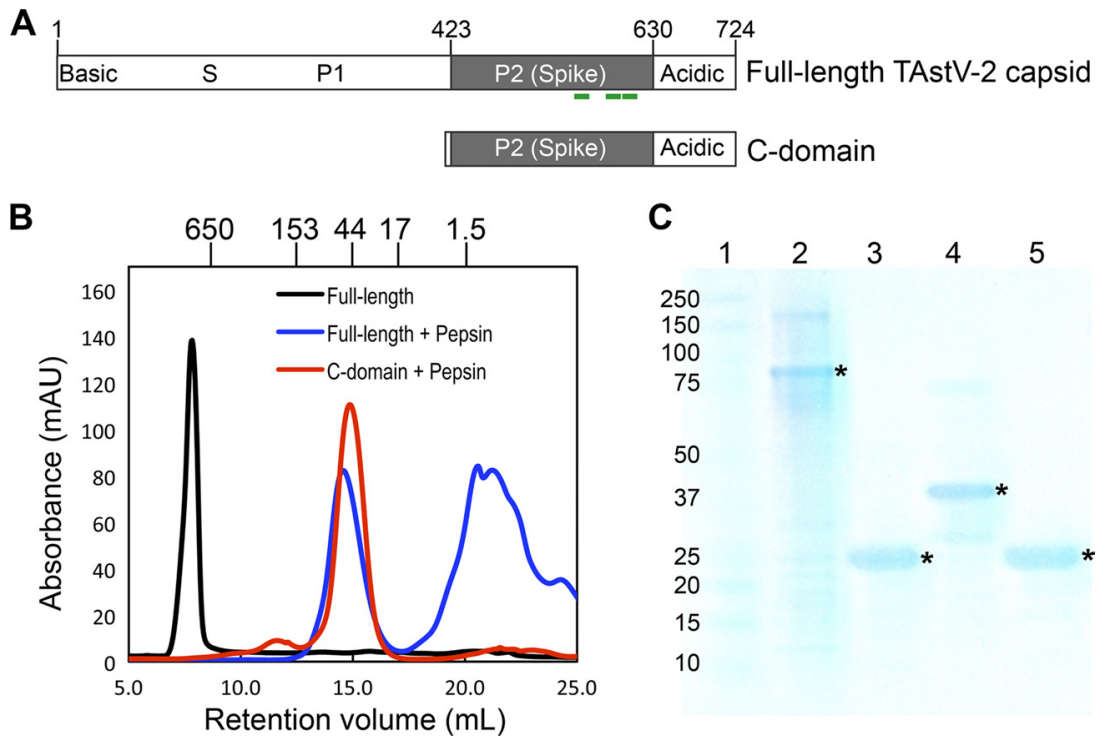

FIG 1 Proteolytic isolation of TAstV-2 capsid spike domain. (A) Schematic of domains in full-length TAstV-2 capsid protein and TAstV-2 C-domain protein. Green lines represent peptides identified by MALDI-TOF/TOF spectrometry. (B) Size-exclusion chromatography analyses of recombinant full-length TAstV-2 capsid (black), full-length TAstV-2 capsid digested with pepsin (blue), and C domain digested with pepsin (red). Retention volumes of gel filtration standards of known sizes $(\mathrm{kDa})$ are shown above the chromatogram. (C) SDS-PAGE analysis of full-length and C-domain proteins alone or digested with pepsin. Lane 1, molecular mass markers $(\mathrm{kDa})$; lane 2, full-length TAstV-2 capsid protein; lane 3, full-length TAstV-2 capsid digested with pepsin; lane 4, C domain; lane 5, C domain digested with pepsin. Asterisks indicate the bands analyzed by MALDI-TOF/TOF spectrometry.

studies with HAstV-8, we found that HAstV-1 infectivity was minimally affected by chondroitinase but not heparinase treatment, suggesting a potential role for particular carbohydrates in infectivity. Unfortunately, given the lack of an in vitro system for $\mathrm{TAstV}-2$, we are currently unable to directly assess the functional role of carbohydrates in TAstV-2 infectivity. In summary, our studies demonstrate that the avian astrovirus spike domain is structurally distinct from the human protein and that further studies are required to understand the role of carbohydrates as putative astrovirus receptors.

\section{MATERIALS AND METHODS}

Full-length TAstV-2 capsid protein production. cDNA corresponding to ORF2, which encodes the full-length TAstV-2 capsid protein (amino acids 1 to 724; GenBank accession number NP_987088), was cloned into the baculovirus transfer vector pFastBacHT-A in frame with an N-terminal 6-histidine purification tag and additional plasmid-encoded residues (MSYYHHHHHHDYDIPTTENLYFQGAMDPE FKGXAEFALEDRKK). Capsid protein was produced with a baculovirus expression system at the St. Jude Children's Research Hospital Protein Production Facility. Bacmid production, transfection, and baculovirus amplification were carried out according to the instructions in the manufacturer's manual (Invitrogen). Suspension cultures of Sf9 insect cells were cultured in SFX-insect serum-free medium to a density of $2 \times 10^{6}$ cells $/ \mathrm{ml}$ and infected at a multiplicity of infection (MOI) of 10 . Cultures were shaken gently for 3 days at $28^{\circ} \mathrm{C}$. Cells pelleted by centrifugation $(2,400 \times g)$ were resuspended in buffer $(50 \mathrm{mM}$ Tris, $\mathrm{pH}$ 7.9, $500 \mathrm{mM}$ $\mathrm{NaCl}$, and $10 \%$ glycerol) and lysed by microfluidization. Cell lysates were clarified by centrifugation $(48,000 \times g)$, and soluble TAstV- 2 capsid was purified from the supernatant by HisTrap metal-affinity chromatography. Purified TAstV-2 capsid $(0.5 \mathrm{mg} / \mathrm{ml})$ was dialyzed into phosphatebuffered saline (PBS), $\mathrm{pH} 7.6$, flash frozen in liquid nitrogen, and stored indefinitely at $-80^{\circ} \mathrm{C}$. Endotoxin levels were below 0.2 endotoxin unit $(\mathrm{EU}) / \mathrm{ml}$ as determined by the Limulus amebocyte lysate assay (Lonza, Walkersville, MD).
TAstV-2 capsid C-domain production. cDNA corresponding to TAstV-2 capsid residues 421 to 724 (C domain) was cloned into pET52b in frame with a C-terminal thrombin cleavage site and a 10-histidine purification tag. Escherichia coli strain BL21(DE3) was transformed with the plasmid, and recombinant C-domain expression was induced with 1 mM IPTG (isopropyl- $\beta$-D-thiogalactopyranoside) at $16^{\circ} \mathrm{C}$ for $16 \mathrm{~h}$. A selenomethionine-substituted $\mathrm{C}$ domain was expressed in E. coli strain B834(DE3) in M9 minimal medium supplemented with selenomethionine. The $\mathrm{C}$ domain was purified from soluble lysates by HisTrap metalaffinity chromatography. The purified C domain $(1 \mathrm{mg} / \mathrm{ml})$ was dialyzed into $10 \mathrm{mM}$ Tris, $\mathrm{pH} 8.0,200 \mathrm{mM} \mathrm{NaCl}$.

Production of TAstV-2 capsid spike domain by digestion with pepsin. Limited proteolytic digestion of the TAstV-2 capsid or $\mathrm{C}$ domain was carried out with pepsin-agarose beads (Thermo Scientific). Approximately $1 \mathrm{mg}$ purified TAstV-2 capsid or $\mathrm{C}$ domain was shaken for $2 \mathrm{~h}$ at $37^{\circ} \mathrm{C}$ with $100 \mu \mathrm{l}$ pepsin-agarose slurry $(\sim 6 \mathrm{U} / \mu \mathrm{l})$ in a buffer of $100 \mathrm{mM}$ sodium acetate, $\mathrm{pH}$ 4.2. The beads were pelleted by centrifugation $(4,000 \times g)$, and the supernatant was filtered through a $0.22-\mu \mathrm{m}$ filter.

Size-exclusion chromatography analysis of TAstV-2 proteins. Fulllength or pepsin-digested TAstV-2 capsid and C-domain proteins were subjected to size-exclusion chromatography on a Superdex 200 column in $10 \mathrm{mM}$ Tris, pH 8.0, and $200 \mathrm{mM} \mathrm{NaCl}$. Gel filtration standards (Bio-Rad) were used to estimate the molecular weights of the TAstV-2 proteins in solution. SDS-PAGE was used to estimate the sizes of the denatured TAstV-2 proteins. Trypsin-digested bands from SDS-PAGE were analyzed by matrix-assisted laser desorption ionization-tandem time of flight (MALDI-TOF/TOF) spectrometry. Purified, pepsin-digested C domain corresponding to the TAstV-2 spike domain was concentrated to $2 \mathrm{mg} /$ $\mathrm{ml}$, flash frozen in liquid nitrogen, and stored indefinitely at $-80^{\circ} \mathrm{C}$.

Crystal structure determination. TAstV-2 capsid spike domain crystals were grown by the hanging-drop vapor diffusion method at $18^{\circ} \mathrm{C}$. The capsid spike domain crystallized in a well solution of $12 \%$ polyethylene glycol 4000 (PEG 4000) and $0.2 \mathrm{M}$ ammonium sulfate. Crystals were quickly transferred to a cryo-solution containing 15\% PEG 4000, $0.2 \mathrm{M}$ ammonium sulfate, and $25 \%$ glycerol before being frozen in liquid nitrogen. Diffraction data were collected at a cryogenic temperature at the 
TABLE 1 Crystallographic statistics

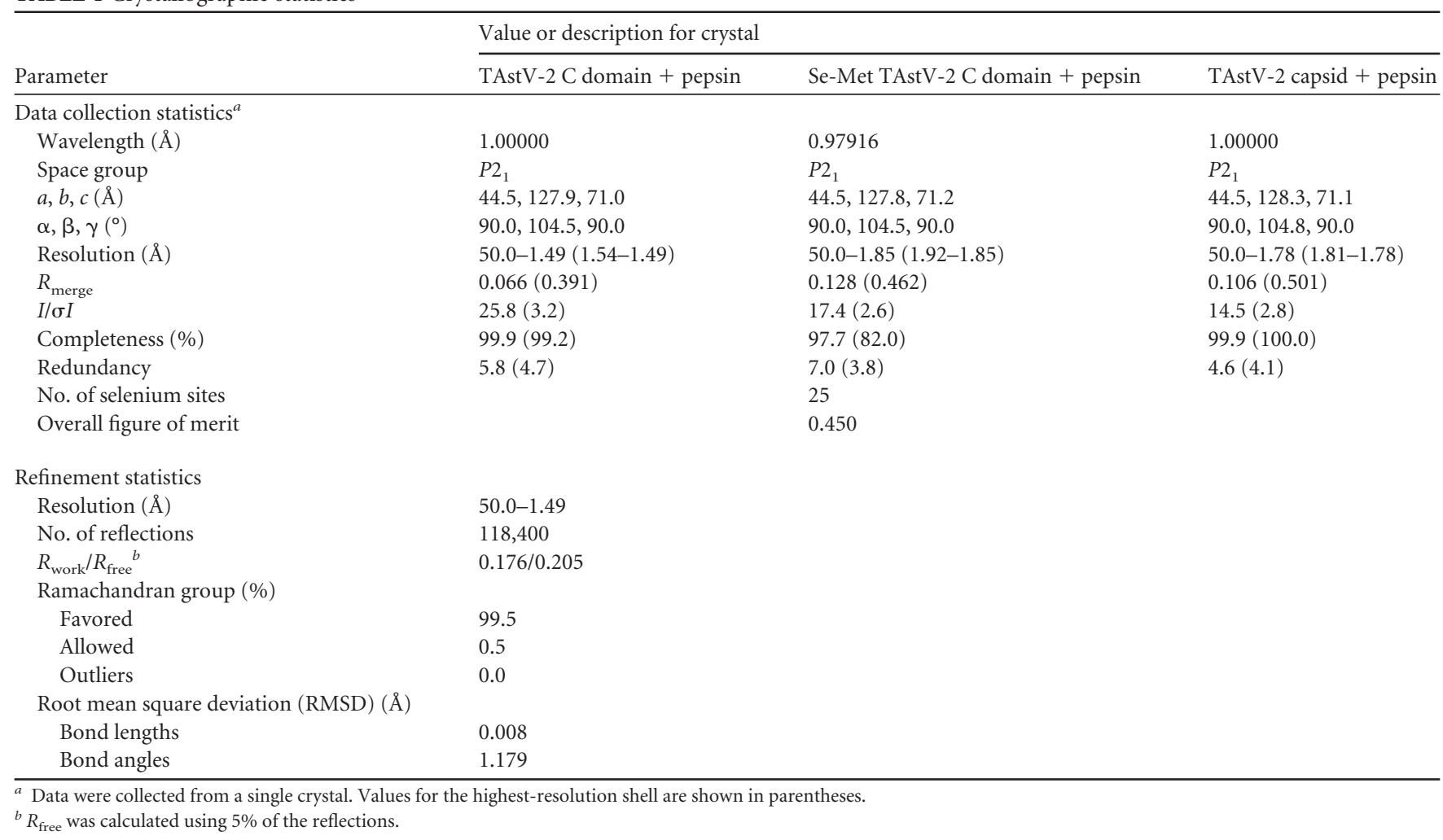

Southeastern Regional Collaborative Access Team's 22-ID and 22-BM beamlines at the Advanced Photon Source (Argonne National Laboratory, Chicago, IL). Data processing and reduction were completed by using HKL-2000 software (35).

The TAstV-2 spike domain structure was solved by single-wavelength anomalous dispersion (SAD). PHENIX (36) was used to determine the locations of 25 selenium sites and to build an initial structural model. Although only 16 possible selenium sites were predicted (16 methionines in the spike domain), 9 additional sites were identified that corresponded to alternate selenomethionine side chain rotamers resolvable at this high resolution. Using the initial model and native data, the structure was refined and rebuilt using Refmac5 (37) and Coot (38), respectively. Refinement was monitored by following the $R_{\text {free }}$ value calculated for a random subset (5\%) of reflections omitted from refinement.

Cell culture and enzyme digestion. Caco- 2 and BHK cells were maintained in Eagle minimum essential medium (MEM; MediaTech) supplemented with 10\% fetal bovine serum (Benchmark), glutaMax-I (Gibco), 1 $\mathrm{mM}$ sodium pyruvate (Gibco), and penicillin-streptomycin (Gibco) at $37^{\circ} \mathrm{C}$ with $5 \% \mathrm{CO}_{2}$. Caco-2 cells were plated in 96- or 12 -well plates and allowed to differentiate for 4 to 6 days. Chondroitinase ABC from Proteus vulgaris (Sigma) was resuspended in PBS with $0.01 \%$ bovine serum albumin (BSA; Gibco), and dilutions were made in MEM containing $50 \mathrm{mM}$ Tris, $\mathrm{pH}$ 8.0, $60 \mathrm{mM}$ sodium acetate, and $0.02 \%$ BSA. Heparinase III from Flavobacterium heparinum (Sigma) was resuspended in PBS containing 20 $\mathrm{mM}$ Tris, $\mathrm{pH} 7.5,4 \mathrm{mM} \mathrm{CaCl}_{2}$, and $0.1 \mathrm{mg} / \mathrm{ml} \mathrm{BSA}$, and dilutions were made in MEM containing 0.3\% BSA. Enzymes or corresponding media alone were added to cells for $2 \mathrm{~h}$ at $37^{\circ} \mathrm{C}$, and a subset of cells were trypsinized and counted using an Invitrogen Countess automated cell counter to determine the number of cells per well and percent viability.

Virus propagation and purification. Differentiated Caco- 2 cells were serum starved in MEM with $5 \mu \mathrm{g} / \mathrm{ml}$ type IV porcine trypsin (Sigma) for $1 \mathrm{~h}$ at $37^{\circ} \mathrm{C}$. HAstV-1 in serum-free MEM with $5 \mu \mathrm{g} / \mathrm{ml}$ porcine trypsin was added for $90 \mathrm{~min}$ at $37^{\circ} \mathrm{C}$, after which the inoculum was removed and replaced with MEM containing $10 \mu \mathrm{g} / \mathrm{ml}$ porcine trypsin. Media and cells were collected after 4 days and sonicated four times for $10 \mathrm{~s}$ each on ice. Virus was purified by banding on a sucrose gradient and then dialyzed in PBS. All viral stocks were quantified using a fluorescent-focus assay to determine the MOI in focus-forming units per $\mathrm{ml}$ (FFU/ml).

Fluorescent-focus assay. Ninety-six-well plates of Caco-2 cells were inoculated with HAstV-1 in MEM containing $0.3 \%$ BSA or in medium alone for $1.5 \mathrm{~h}$ at $37^{\circ} \mathrm{C}$, at which time the virus was replaced with MEM containing $0.3 \%$ BSA. After 16 to $24 \mathrm{~h}$, cells were fixed in $4 \%$ paraformaldehyde for $20 \mathrm{~min}$ at room temperature. Cells were washed with PBS, permeabilized with $0.5 \%$ (vol/vol) Triton X-100 in PBS for $15 \mathrm{~min}$, and then blocked in 5\% normal goat serum in PBS for $1 \mathrm{~h}$ at room temperature. Cells were stained with the HAstV monoclonal antibody clone 8E7 and with secondary anti-mouse IgG-Alexa 488 (Invitrogen) at a 1:100 dilution for $1 \mathrm{~h}$ at room temperature. DAPI (4',6-diamidino-2-phenylindole; Sigma) was used to counterstain nuclei. Wells were imaged on an Evos microscope (Advanced Microscopy Group) at a magnification of $\times 40$, using identical parameters for each treatment. Nuclei and fluorescein isothiocyanate-positive $\left(\mathrm{FITC}^{+}\right)$cells were counted using ImageJ software.

Flow cytometry. Twelve-well plates of Caco-2 and BHK cells were precooled on ice after enzyme digestion. Purified HAstV-1 labeled with Dylight 488 N-hydroxysuccinimide (NHS)-ester fluorophores (Thermo Scientific) according to the manufacturer's protocol was added to cells and allowed to bind for $1 \mathrm{~h}$ on ice. Unbound virus was removed, and cells were trypsinized, fixed with $2 \%$ paraformaldehyde for $20 \mathrm{~min}$, and resuspended in PBS containing 5\% BSA after washing. Stained cells were acquired on a FACSCalibur flow cytometer (BD Biosciences) and analyzed on FlowJo software (Tree Star). FITC ${ }^{+}$gates were set based on cells alone.

Statistics. The statistical significance of the data was determined by using the 2-tailed Student $t$ test in Microsoft Excel. All assays were run in triplicate, and data are representative of at least two separate experiments. 

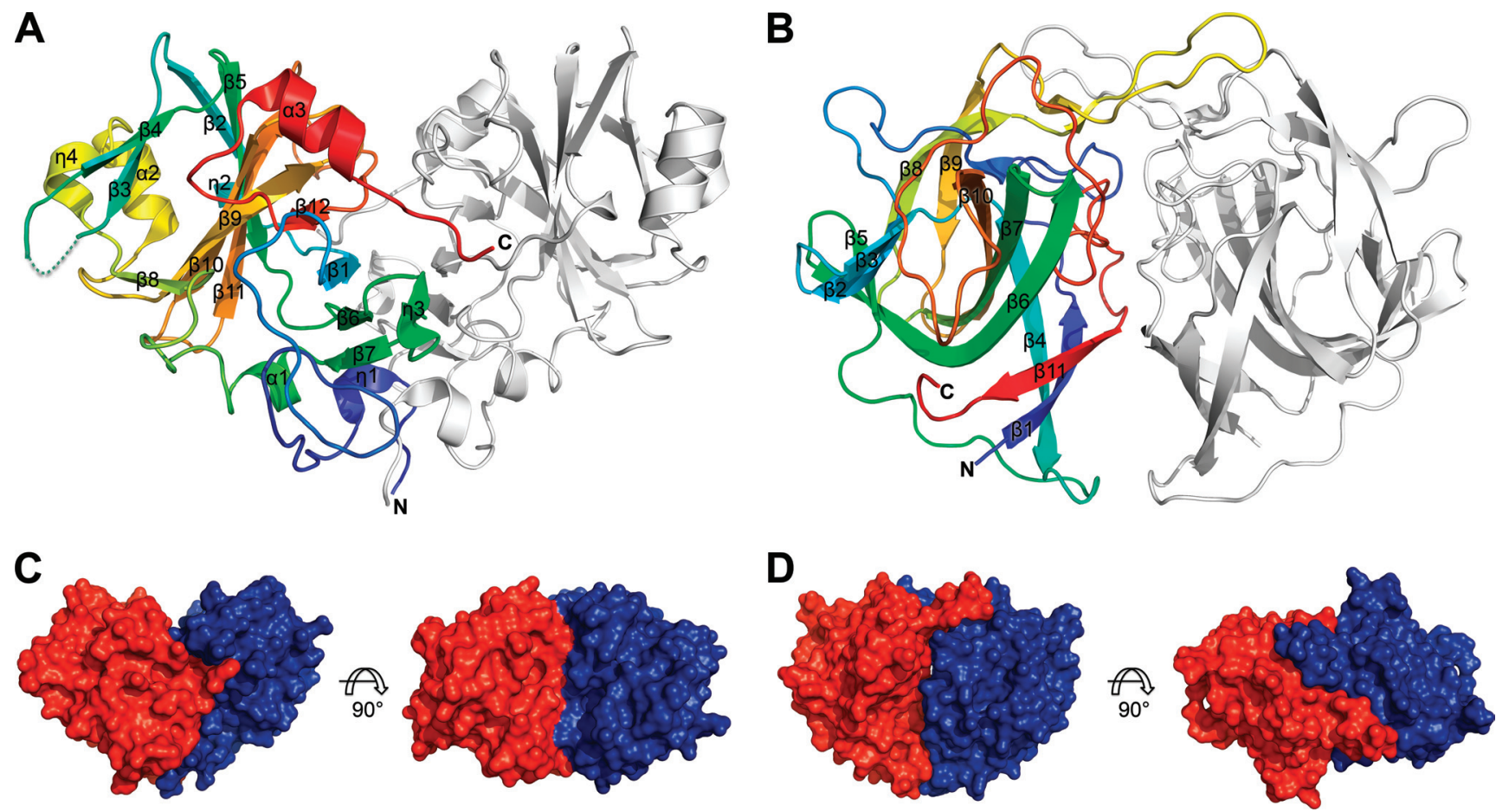

FIG 2 Crystal structure of the TAstV-2 spike domain and comparison to the HAstV-8 spike domain. (A) Side view of the TAstV-2 spike domain, shown as a cartoon representation. One subunit is gray, and the other is rainbow colored from the $\mathrm{N}$ terminus (blue) to the $\mathrm{C}$ terminus (red). (B) Side view of the HAstV-8 spike domain (PDB entry 3QSQ), displayed in the same orientation and with the same coloring as in panel A. (C) Surface representations of the TAstV-2 spike domain, shown as side and top views, respectively. One dimer subunit is colored red, and the other is colored blue. (D) Equivalent surface representations of the HAstV-8 spike domain.

Error bars represent standard deviations, and statistical significance was defined as having a $P$ value of $<0.05$.

Protein structure accession number. The TAstV-2 spike domain structural model and structure factors have been deposited into the online Protein Data Bank (PDB; www.pdb.org) as PDB entry 3TS3.

\section{RESULTS}

Isolation of TAstV-2 spike domain. The astrovirus capsid is a multidomain protein (Fig. 1A), and we wished to isolate TAstV-2 capsid fragments for structural studies. We first carried out limited proteolytic digestion of the TAstV-2 capsid protein with pepsin and analyzed the proteolytic products by size-exclusion chromatography and SDS-PAGE. Undigested, full-length recombinant TAstV-2 capsid protein is an $85-\mathrm{kDa}$ protein as visualized by SDS-PAGE, but it eluted in the void volume of the size-exclusion chromatography column (apparent molecular mass of $>650 \mathrm{kDa}$ ) (Fig. $1 \mathrm{~B}$ and C). This suggests that TAstV-2 capsids form multimers such as capsomers or virus-like particles (VLPs), consistent with the formation of VLPs by recombinant HAstV-1 capsid (39-42). Pepsin digestion yielded a $26-\mathrm{kDa}$ stable fragment (Fig. 1C) that eluted from the size-exclusion chromatography column at an apparent molecular mass of $\sim 60 \mathrm{kDa}$ (Fig. 1B), suggesting that the fragment forms a dimer in solution. In-gel tryptic digestion and mass spectrometric analyses of the $26-\mathrm{kDa}$ band identified peptides in the C-terminal region of the capsid protein (Fig. $1 \mathrm{~A})$, and this established that the fragment corresponds to the C-terminal spike domain, which forms a dimeric protrusion on the surface of the astrovirus capsid. No other significant
TAst $\mathrm{V}-2$ capsid fragments of $>5 \mathrm{kDa}$ were produced by pepsin digestion.

To produce the isolated spike domain of the TAstV-2 capsid protein, we expressed the $\mathrm{C}$-terminal region ( $\mathrm{C}$ domain; residues 421 to $724 ; 35 \mathrm{kDa}$ ) in E. coli and found that it was partially soluble and could be purified (Fig. 1A and C). Pepsin digestion of the $\mathrm{C}$ domain also yielded a $26-\mathrm{kDa}$ domain (Fig. 1C) that eluted from the size-exclusion chromatography column at an apparent molecular mass of $\sim 60 \mathrm{kDa}$ (Fig. 1B), and this suggested that the same domain was proteolytically released from both the full-length TAstV-2 capsid and the C domain. Structural studies (see below) allowed us to identify the $26-\mathrm{kD}$ fragment as the astrovirus capsid "spike domain."

Crystal structure of TAstV-2 capsid spike domain. To gain insights into the function of the spike domain, we used X-ray crystallography to determine its high-resolution structure. The purified TAstV-2 spike domains obtained by pepsin digestion of full-length capsid or the $\mathrm{C}$ domain both yielded identical crystal forms (Table 1), supporting our biochemical evidence that they are the same domains. During the course of our studies, the crystal structure of the HAstV-8 spike domain was published (23), but attempts to use this structure to solve the TAstV-2 spike domain structure by molecular replacement failed. This suggested that the TAstV-2 and HAstV-8 spike domains have quite different structures, consistent with the lack of identifiable sequence homology. We therefore generated a selenomethionine-substituted sample and used SAD methods to determine its structure to $1.5-\AA$ resolution (Fig. 2A and Table 1). The structure of the TAstV-2 spike 

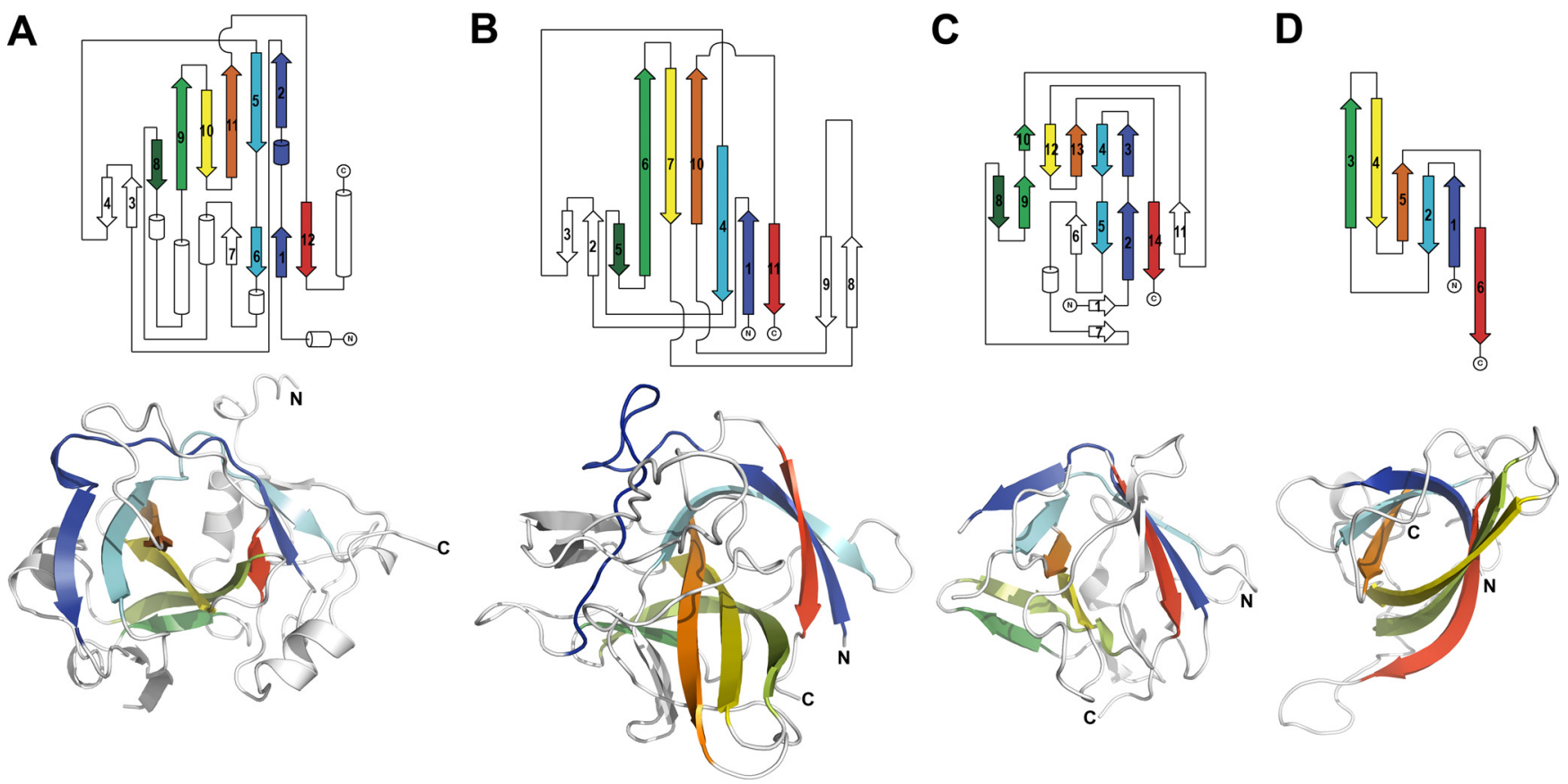

FIG 3 Structural comparison of TAstV-2, HAstV-8, HEV, and norovirus spike domains. (A to D) Topology diagrams (top) and cartoon representations (bottom) of the TAstV-2, HAstV-8, HEV, and norovirus (PDB entries 3TS3, 3QSQ, 3HAG, and 2OBS, respectively) spike domains, respectively. The core $\beta$-barrel strands are colored blue, cyan, light green, yellow, orange, and red. In HAstV-8, TAstV-2, and HEV, the additional structurally conserved $\beta$-strand that packs alongside the $\beta$-barrel is colored dark green. Remaining structural elements are shown in white. In the cartoon representations, only one dimer subunit is shown and is structurally aligned to the TAstV-2 spike domain.

domain (residues 423 to 630 ) reveals a tight dimeric protein, consistent with the size-exclusion chromatography data. The structural scaffold of each protomer comprises an antiparallel $\beta$-barrel (strands $\beta 1, \beta 2, \beta 5, \beta 6, \beta 8, \beta 9, \beta 10$, and $\beta 11$ ) with a tightly packed hydrophobic core. The $\beta$-barrel is capped at one end by strand $\beta 7$ and helix $\alpha 1$, which insert hydrophobic residues into the $\beta$-barrel hydrophobic core. The $\beta$-barrel is further flanked by two additional $\alpha$-helices ( $\alpha 2$ and $\alpha 3$ ), four 3/10 $\eta$-helices, and a $\beta$-hairpin composed of strands $\beta 3$ and $\beta 4$. Note that the topology of the $\beta$-barrel strands is structurally distinct from the "jelly-roll fold" or "lectin fold" that is often found in virus surface proteins.

As anticipated, the structure of the TAstV-2 spike is strikingly different from the HAstV-8 structure (Fig. 2). The TAstV-2 spike is an $\alpha / \beta$ protein, whereas the HAst $V-8$ spike is mostly $\beta$. In terms of overall shape, the TAstV-2 spike resembles a tightly packed heart, whereas the HAstV-8 spike is bowl-like, with a hole in the middle (Fig. 2C and D). Structural analysis by the PISA server (43) revealed that the TAstV-2 spike dimer interface is $\sim 4,600 \AA^{2}$ and is mediated by 121 interface residues, whereas the HAstV- 8 spike dimer interface is $\sim 3,500 \AA^{2}$ and is mediated by 103 interface residues.

Comparison with HAstV-8 capsid spike domain and other viral capsid spike domains. Despite the significant structural and morphological differences between the HAstV-8 and TAstV-2 spike domains, closer inspection of the folding topology does suggest a distant relationship to the HAstV-8 spike as well as to the spike domains of other enteric virus capsid proteins, including those of HEV and norovirus (27-30, 32, 33, 44) (Fig. 3 and Table 2 ). All have a core $\beta$-barrel structure in which the $\beta$-strands have a common pattern of connectivity (Fig. 3). Their structural divergence results from the considerable variability in the lengths of the $\beta$-strands within the $\beta$-barrels and intervening structural elements. The TAstV-2, HAstV-8, and HEV spike domains all have an additional structurally conserved $\beta$-strand (strand $\beta 8$ in TAstV-2) that packs alongside and, in the case of TAstV-2, extends the $\beta$-barrel (Fig. 3A, B, and C, dark green arrows). Unique to the astrovirus spike domains are a $\beta$-hairpin insertion (strands $\beta 3$ and $\beta 4$ in TAstV-2) that packs alongside the $\beta$-barrel (Fig. 3). Surprisingly, structural alignments of these spike domains by use of the DaliLite server (45) revealed that the TAstV-2 domain is most closely related to the HEV domain, with a $Z$ score of 4.3 , compared to 3.1 for the HAstV-8 comparison (Table 2). This appears to be due to several common structural features that include shorter and more discontinuous $\beta$-barrel strands, similar structural

TABLE 2 DaliLite scores comparing viral capsid spike domains ${ }^{a}$

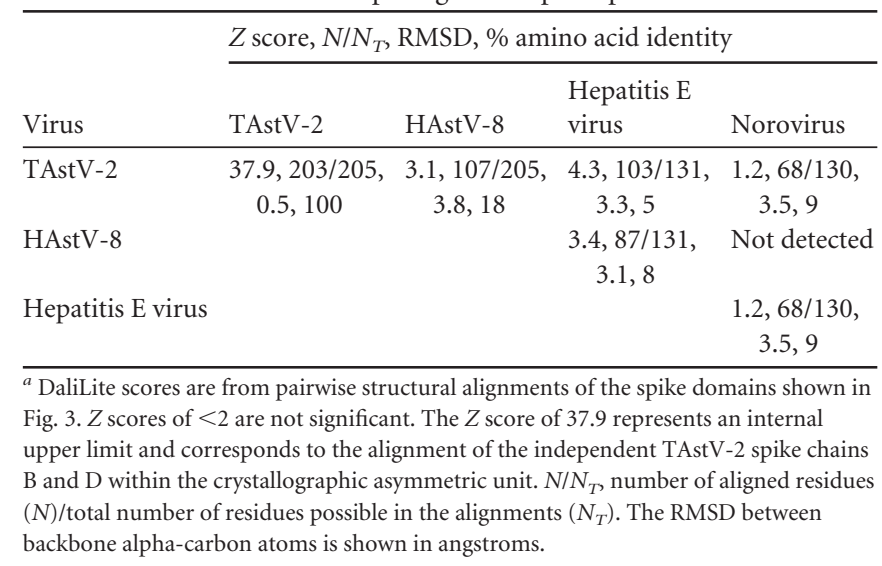



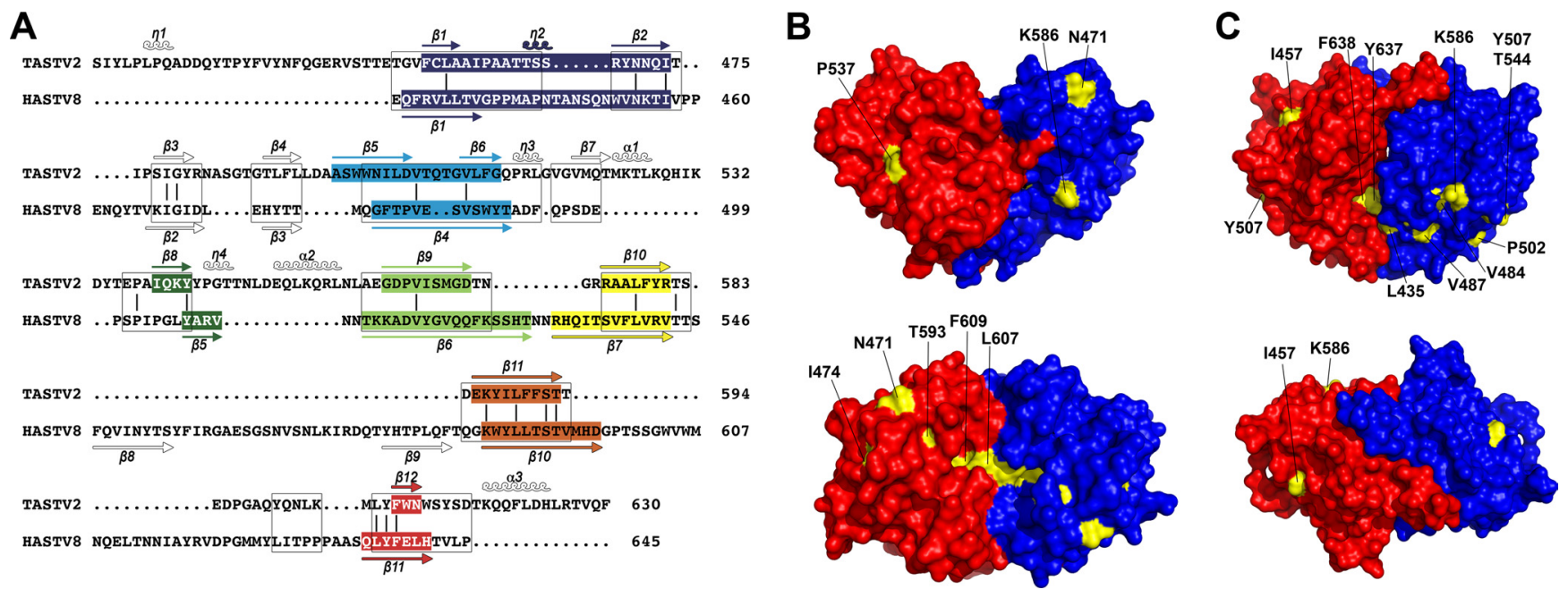

FIG 4 Structural alignment of TAstV-2 and HAstV-8 spike domains. (A) Structural pairwise sequence alignment of the TAstV-2 and HAstV-8 spike domains by use of the DaliLite server. Structural elements are colored as described in the legend to Fig. 3. Structurally equivalent residues are boxed. Identical amino acids in structurally equivalent locations are noted by vertical lines between the sequences. (B and C) Surface representations of the TAstV-2 and HAstV-8 spike domains, respectively, shown as side and top views. One dimer subunit is colored red, and the other is colored blue. Identical amino acids in structurally equivalent locations are colored yellow and labeled.

insertions (strand $\beta 7$ and helix $\alpha 1$ in TAstV-2), and additional structural elements at the $\mathrm{N}$ terminus (Fig. 3). These structural data support recent evidence that HEV and astroviruses have a much closer phylogenetic relationship than previously expected $(23,26)$.

A major difference between the TAstV-2 and HAstV-8 domains is a $\beta$-hairpin insertion in the latter (strands $\beta 8$ and $\beta 9$ ) that contains a long intervening loop (Fig. 4A). In the HAstV-8 spike dimer, these two loops form reciprocal "crossing arms" that form intimate interactions across the top of the dimer that are thought to play a key role in dimer formation (Fig. 3B and D) (23). This insertion contains a number of conserved residues that may be involved in the formation of a putative HAstV receptor-binding pocket (23). The absence of this structural insertion in the TAstV-2 domain leads not only to a dramatically different overall morphology (Fig. 3) but also to the lack of an equivalent putative HAstV receptor-binding pocket. This suggests that the two viruses have completely different receptor-binding sites, which is supported by a structural alignment of the TAstV-2 and HAstV-8 domains (Fig. 4A) that revealed no patches of identical, surfaceexposed residues that might hint of a conserved receptor-binding site (Fig. 4B and 5C).

Identification of putative avian receptor-binding site(s). To identify a putative receptor-binding site on our TAstV-2 spike structure, we carried out sequence alignments of several avian astrovirus capsid spike domains and mapped the conserved residues onto the structure (Fig. 5 and 6). Alignment with two other members of the Avastrovirus I genogroup, TAstV-3 and duck astrovirus I (DAstV-1), gave identities of $74 \%$ and $51 \%$, respectively (Fig. 5A), suggesting that they all have very similar structures. When mapped onto the structure, the pattern of conservation reveals a putative receptor-binding site (site I) in a shallow groove at the top edge of the spike (Fig. 5B, purple circles), containing the conserved residues R469, N471, W498, R556, D596, and Y601 (Fig. 5A, B, and C). The presence of an exposed tryptophan and several charged residues is intriguing, because these types of resi- dues are typically found in carbohydrate-binding pockets $(32,33$, $46,47)$. A sulfate ion derived from the crystallization solution is observed at site I, bound to the conserved R556 on helix $\alpha 2$, and this may reflect the binding pocket for a negatively charged moiety on the receptor. Deletion of a nearby residue (Q552) on helix $\alpha 2$ (Fig. 5A and B, orange stars) was recently associated with an increased incidence of disease in turkeys infected with TAstV-2 (48), and it is possible that this deletion facilitates site I interactions with the host receptor.

The structural similarity to the norovirus spike domain noted above prompted us to investigate the TAstV-2 spike structure at regions equivalent to the norovirus receptor-binding sites. Norovirus binds histo-blood group antigen trisaccharides by use of two distinct binding pockets, depending on the norovirus genogroup (33). Structural alignment of the TAstV-2 and norovirus spikes revealed two interesting sites: sites II and III (Fig. 5B, green and cyan circles, respectively). Site II comprises a narrow but pronounced pocket $\left(\sim 200 \AA^{3}\right)$ at the top of the structure, containing four partially or completely conserved hydrophobic residues at the base (F591, L604, M606, and F609) and two conserved hydrophilic residues at the rim (D570 and R574) (Fig. 5A, B, and D). This site was also identified as the top hit by the software ICM-Pro to identify surface cavities. Site III is situated on the side of the TAstV-2 spike and comprises a large patch of conserved residues (Y542, P544, K554, L557, N558, L559, and E561) (Fig. 5A, B, and E). Site III is also adjacent to residue 552 , which was associated with an increased incidence of disease in turkeys infected with TAstV-2 (48) (Fig. 5A and B, orange stars).

Finally, an alignment of the TAstV-2 spike domain sequence with members of the Avastrovirus II genogroup, TAstV-1 and avian nephritis viruses 1 and 2 (ANV1 and -2), revealed identities of between 10 and 16\% (Fig. 6). Despite the low level of sequence conservation, the alignments with respect to the secondary structure suggest that the Avastrovirus II spike domains have similar structures to that of the TAstV-2 spike. The largest difference is an apparent 7-residue deletion at $\mathrm{TAstV}-2$ spike residues 482 to 488 , 

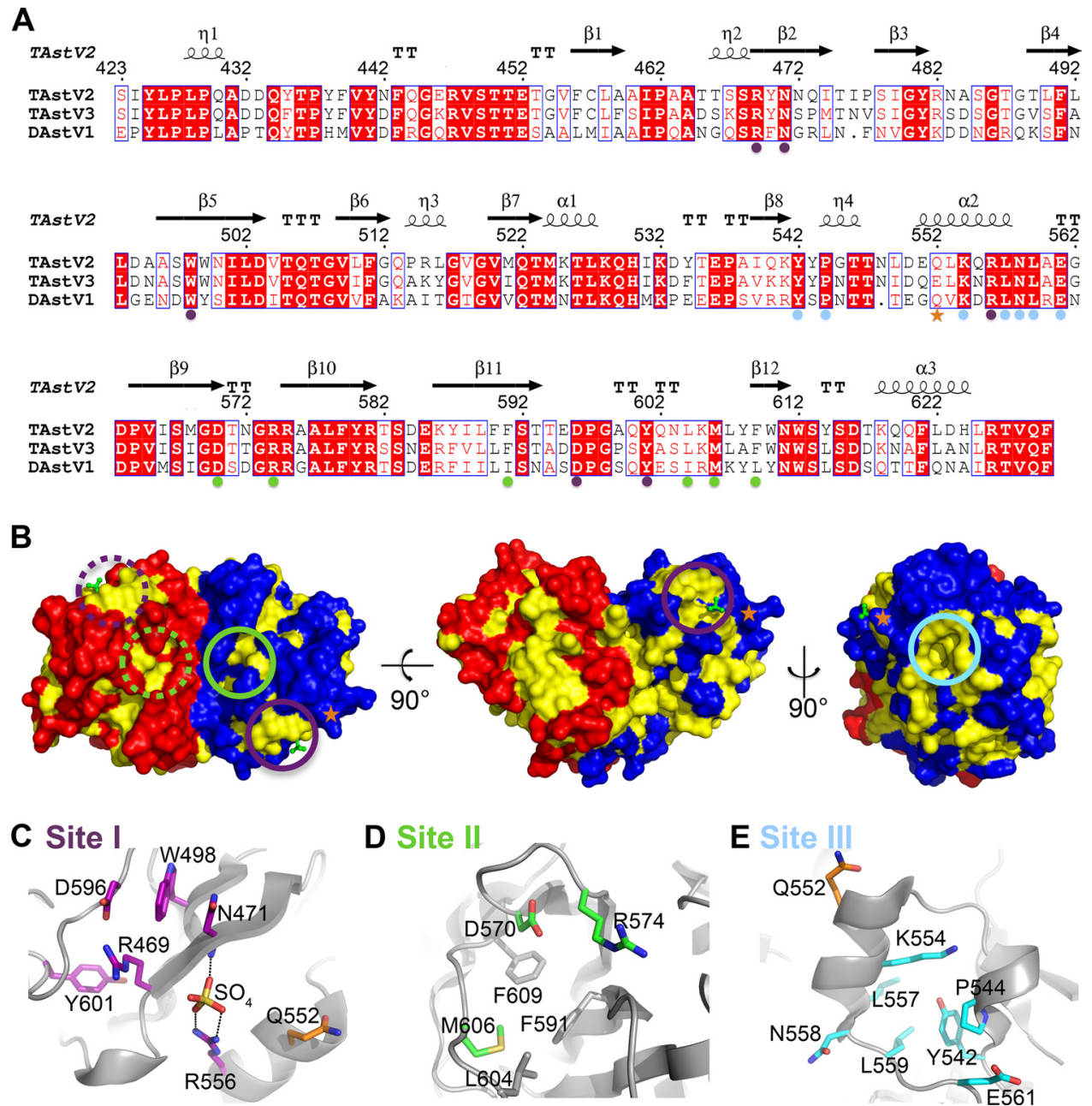

FIG 5 Conserved spike domain residues in Avastrovirus I genogroup astroviruses. (A) Multiple sequence alignment of representative Avastrovirus I genogroup capsid spike domains, for TAstV-2, TAstV-3, and DAstV-1 (GenBank accession no. Q9Q3G5, Q5UAH3, and D8L641, respectively), using the online server ESPript. Conserved residues are shown in white with a red background, and similar residues are shown with red type. Colored dots indicate the residues on the surface of the TAstV-2 spike domain that are circled in panel B (in the same color). An orange star locates residue 552, which may affect disease outcomes in turkeys infected with TAstV-2 (48). (B) Surface representation of the TAstV-2 spike domain, shown as top, side, and alternate side views (left, middle, and right panels, respectively). One dimer subunit is colored red, and the other is colored blue. Identical amino acids as found in panel A are colored yellow. Site I is circled in purple, site II is circled in green, and site III is circled in cyan. The 2 -fold related sites in the other dimer subunit are denoted by broken circles. The sulfate ion within site I observed in the crystal structure is shown as a ball-and-stick model and colored green. (C to E) Detailed views of sites I, II, and III. The carbon atoms of conserved residues are colored according to their site locations as shown in panels A and B. Residue 552 is colored orange. Nonconserved residues (site II) are colored gray.

suggesting that Avastrovirus II spike domains may have a shorter $\beta 3-\beta 4$ hairpin (Fig. 6). However, when the Avastrovirus I and II capsid spike domains were aligned, only 17 residues were found to be conserved strictly, and none of them clustered together when mapped onto the TAstV-2 capsid spike structure (Fig. 6). These data suggest that although the Avastrovirus II capsid spikes are likely structurally similar to that of TAstV-2 (Avastrovirus I), they may have a distinct receptor-binding site.

Role of carbohydrates in HAstV-1 infectivity. In the structure of the TAstV-2 spike, we observed a patch of surface-exposed, conserved residues (site I) with molecular characteristics of a carbohydrate-binding pocket. In addition, the HAstV-8 spike domain structure revealed a putative carbohydrate-binding pocket, and it was further reported that heparin, heparan sulfate, or dextran sulfate was able to reduce HAstV-8 infectivity in Caco- 2 cells
(23). Unfortunately, there is no system to study TAstV-2 infectivity in vitro, and attempts to isolate turkey primary intestinal epithelial cells that bind to TAstV-2 have been unsuccessful. Thus, to determine if glycans were required for astrovirus binding and/or infection, we pretreated Caco-2 cells with heparinase or chondroitinase $\mathrm{ABC}$ to remove heparin or chondroitin sulfates, respectively, and purified FITC 488-labeled HAstV-1 was added to the cells on ice to allow the virus to bind but not endocytose. Unbound virus was removed, cells were fixed, and the percentage of FITC-positive cells was determined by flow cytometry. We found that neither chondroitinase nor heparinase treatment significantly decreased HAstV-1 binding to Caco-2 cells (Fig. 7A). However, we saw a small but consistently significant $(\sim 25 \%)$ decrease in HAstV-1 infection in cells treated with 4 or $10 \mathrm{U} / \mathrm{ml}$ chondroitinase (Fig. 7B). A further increase in the chondroitinase concen- 

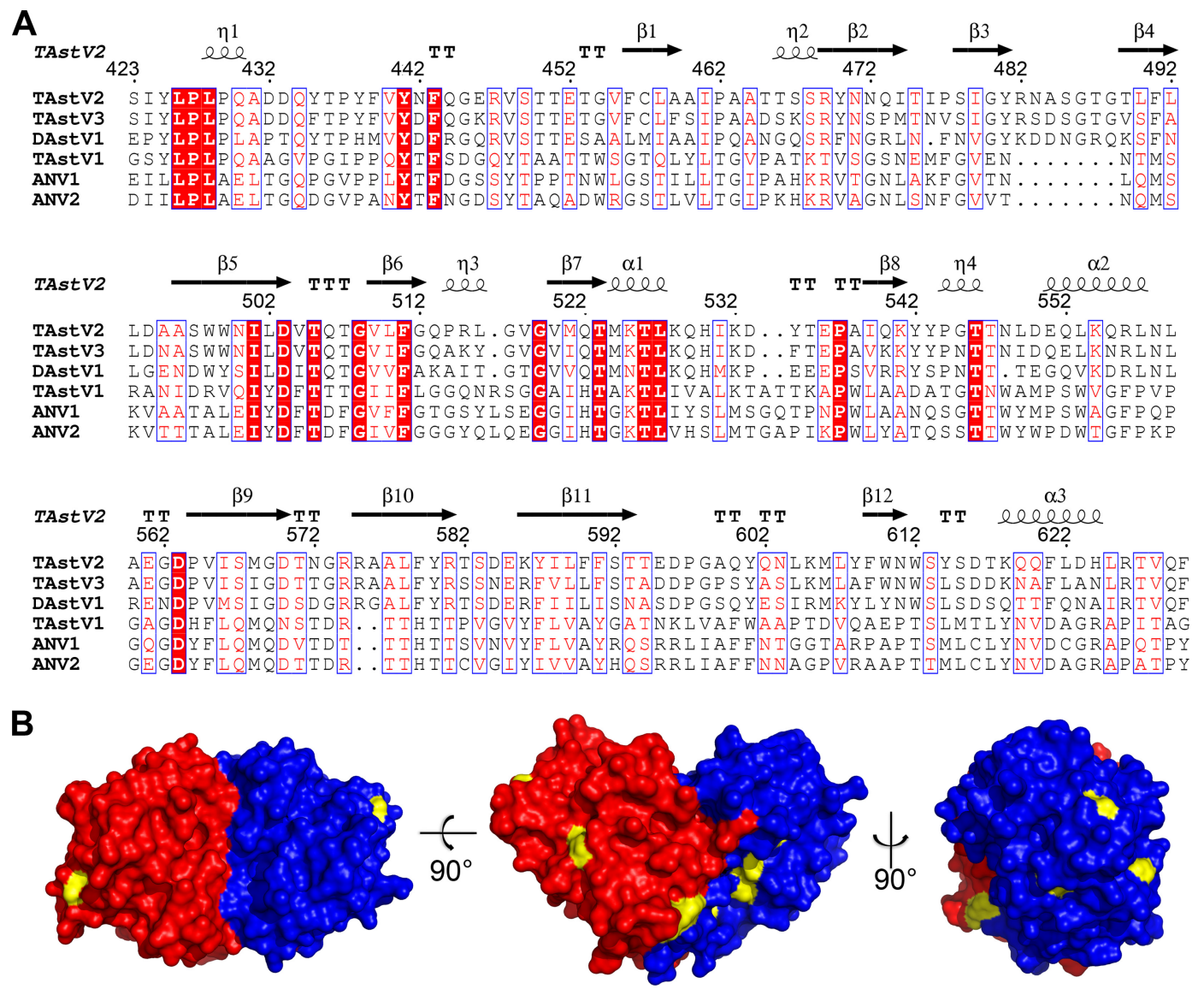

FIG 6 Conserved spike domain residues in Avastrovirus I and II genogroup astroviruses. (A) Multiple sequence alignment of representative Avastrovirus I and II genogroup capsid spike domains: TAstV-2 (GenBank accession no. Q9Q3G5), TAstV-3 (GenBank accession no. Q5UAH3), DAstV-1 (GenBank accession no. D8L641), TAstV-1 (GenBank accession no. Q9JH68), avian nephritis virus 1 (ANV1; GenBank accession no. Q9JGF1), and ANV2 (GenBank accession no. Q99IE8). Conserved residues are shown in white with a red background, and similar residues are shown with red type. (B) Surface representation of the TAstV-2 spike domain, shown as top, side, and alternate side views (left, middle, and right panels, respectively). One dimer subunit is colored red, and the other is colored blue. Identical amino acids as found in panel A are colored yellow.

tration resulted in decreased cell viability (data not shown). We did not observe the same decrease in infection with cells pretreated with heparinase. Additional studies are needed to identify how carbohydrates are involved in astrovirus infection, as well as if other glycans play a role in cellular binding and entry.

\section{DISCUSSION}

We report here the 1.5- $\AA$-resolution crystal structure of the TAstV-2 capsid spike domain. By comparison with the HAstV-8 capsid spike domain and other viral spike domains, we provide new molecular insights into astrovirus host cell entry, species specificity, and evolution.

A critical step in species specificity is the initial interaction between a virus and host cell, and changes in receptor binding can play a role in cross-species transmission. Often, only a few mutations are sufficient to change receptor-binding specificity in a virus attachment protein, with few or no structural changes. One example of this is the well-studied influenza virus hemagglutinin protein, which has the same structural fold in avian and human influenza viruses but has different residues in the sialic acid receptor-binding pocket. Recently, it was shown that as few as four mutations in the hemagglutinin protein can convert avian to hu- man receptor-binding specificity and also confer transmission of avian $\mathrm{H} 5 \mathrm{~N} 1$ influenza viruses to a mammalian host $(49,50)$. Unlike the influenza virus hemagglutinin protein, the human and avian astrovirus capsid spike domains have striking differences in sequence, structural fold, and overall morphology. Although the presence of a core $\beta$-barrel structure in both the avian and human astrovirus capsid spike domains allowed us to structurally align them, there were no patches of identical, surface-exposed residues to support the existence of a conserved receptor-binding site. Assuming that the astrovirus capsid spike domain contains the only astrovirus receptor-binding site, these data suggest that conversion of avian to human receptor-binding specificity would require major sequence and structural changes in the capsid spike domain. However, despite evidence that the astrovirus capsid spike domain alone contains the receptor-binding site $(15,17)$, we cannot rule out the possibility that another region of the astrovirus capsid contains receptor-binding properties that may more easily convert avian to human receptor-binding capabilities.

In contrast, our data do support the hypothesis that transmission between avian species is likely. Our predicted structural similarity in all avian capsid spike domains suggests that one or more mutations may be sufficient to compose a receptor-binding 

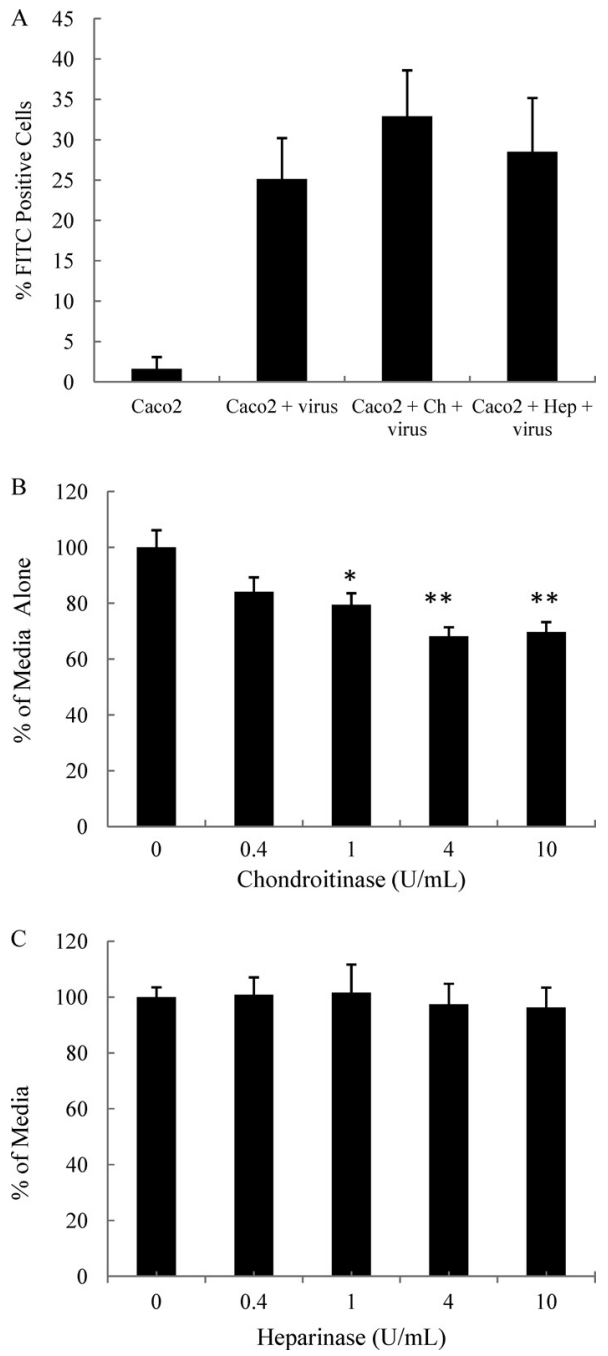

FIG 7 Effect of cell surface deglycosylation on HAstV-1 binding and infection. Caco-2 cells were incubated with $4 \mathrm{U} / \mathrm{ml}$ (A) or increasing concentrations (B and C) of chondroitinase ( $\mathrm{Ch}$ ), heparinase III (Hep), or medium alone for $2 \mathrm{~h}$. (A) Precooled cells were inoculated with FITC 488-labeled HAstV-1 at an MOI of $0.35 \mathrm{FFU} / \mathrm{cell}$ for $1 \mathrm{~h}$ on ice. Unbound virus was removed from cells prior to fixing. The percentage of FITC-positive cells was determined by flow cytometry. (B and C). Cells were inoculated with HAstV- 1 at an MOI of $0.5 \mathrm{FFU} /$ cell, and the percentage of FITC-positive cells was determined by a fluorescentfocus assay as described in Materials and Methods. Data are means plus standard deviations and are representative of 2 independent experiments. ${ }^{*}, P<$ $0.5 ;{ }^{* *}, P<0.005$.

pocket for a new avian species. There is already evidence for avianto-avian astrovirus transmission between turkeys and ducks (6) or guinea fowl $(51,52)$ and between chickens and pigeons $(53)$, water fowl (54), or ducks (55).

A major gap in astrovirus biology is the identity of the host cell receptor(s). The observation of conserved patches of residues on the surfaces of both human and avian astrovirus capsid spike domains with molecular characteristics of a carbohydrate-binding pocket prompted us to further investigate the affinity of $\mathrm{HAstV}-1$ for select carbohydrates by flow cytometry and infectivity studies. In contrast to the data with $\mathrm{HAstV}-8$, we found no role for heparin in $\mathrm{HAstV}-1$ binding or infectivity. In contrast, although there was no obvious impact on binding by flow cytometry, pretreating Caco-2 cells with chondroitinase led to a minimal, although significant, decrease $(25 \%)$ in HAstV-1 infectivity. Unfortunately, we do not currently have an in vitro system to test the role of carbohydrates in TAstV-2 infectivity. Future studies will explore the interaction of specific carbohydrates with the intact virion, capsid, and spike by use of a glycan array. An alternative possibility is developing reagents to test chicken astrovirus infectivity of avian cells. Regardless, we cannot rule out the possibility that there may be an avidity effect due to the presence of several spike domains on the astrovirus capsid surface that may collectively support binding to host cell carbohydrates.

Finally, our data further support recent evidence that astroviruses are phylogenetically related to HEV. We find that the TAstV-2 capsid spike domain is structurally related to the HEV spike domain, as was found with the HAstV-8 spike domain (23). Dong et al. also described that the HEV capsid has sequence similarity and predicted structural homology to the conserved N-terminal region of the astrovirus capsid protein which forms the capsid shell (23). These data are further supported by the electron microscopy structure of the HAstV capsid, which revealed striking similarities in size, shape, and architecture to the HEV particle (26).

Overall, comparative structural studies such as this one provide new molecular insights into astrovirus host cell entry and help us to understand the potential threat for cross-species transmission of this important family of viruses.

\section{ACKNOWLEDGMENTS}

We thank the Hartwell Center for DNA synthesis, DNA sequencing, protein production, and mass spectrometry experiments.

Funding for this research was provided by the Children's Infection Defense Center, the Hartwell Foundation, and the American Lebanese Syrian Associated Charities at St. Jude Children's Research Hospital.

Data were collected at the Southeast Regional Collaborative Access Team (SER-CAT) 22-ID beamline at the Advanced Photon Source, Argonne National Laboratory. Supporting institutions may be found at www.ser-cat.org/members.html. Use of the Advanced Photon Source was supported by the U.S. Department of Energy, Office of Science, Office of Basic Energy Sciences, under contract W-31-109-Eng-38.

\section{REFERENCES}

1. Glass RI, Noel J, Mitchell D, Herrmann JE, Blacklow NR, Pickering LK, Dennehy P, Ruiz-Palacios G, de Guerrero ML, Monroe SS. 1996. The changing epidemiology of astrovirus-associated gastroenteritis: a review. Arch. Virol. 12(Suppl):287-300.

2. Mendez E, Arias CF. 2007. Astroviruses, p 982-1000. In Knipe DM, Howley PM, Griffin DE, Lamb RA, Martin MA, Roizman B, Straus SE (ed), Fields virology, 5th ed. Lippincott Williams \& Wilkins, Philadelphia, PA.

3. Walter JE, Mitchell DK. 2003. Astrovirus infection in children. Curr. Opin. Infect. Dis. 16:247-253.

4. Burbelo PD, Ching KH, Esper F, Iadarola MJ, Delwart E, Lipkin WI, Kapoor A. 2011. Serological studies confirm the novel astrovirus HMOAstV-C as a highly prevalent human infectious agent. PLoS One 6:e22576. doi:10.1371/journal.pone.0022576.

5. De Benedictis P, Schultz-Cherry S, Burnham A, Cattoli G. 2011. Astrovirus infections in humans and animals-molecular biology, genetic diversity, and interspecies transmissions. Infect. Genet. Evol. 11:1529-1544.

6. Fu Y, Pan M, Wang X, Xu Y, Xie X, Knowles NJ, Yang H, Zhang D. 2009. Complete sequence of a duck astrovirus associated with fatal hepatitis in ducklings. J. Gen. Virol. 90:1104-1108.

7. Imada T, Yamaguchi S, Mase M, Tsukamoto K, Kubo M, Morooka A. 2000. Avian nephritis virus (ANV) as a new member of the family Astro- 
viridae and construction of infectious ANV cDNA. J. Virol. 74:84878493.

8. Koci MD, Seal BS, Schultz-Cherry S. 2000. Molecular characterization of an avian astrovirus. J. Virol. 74:6173-6177.

9. Reynolds DL, Saif YM. 1986. Astrovirus: a cause of an enteric disease in turkey poults. Avian Dis. 30:728-735.

10. Schultz-Cherry S, Kapczynski DR, Simmons VM, Koci MD, Brown C, Barnes HJ. 2000. Identifying agent(s) associated with poult enteritis mortality syndrome: importance of the thymus. Avian Dis. 44:256-265.

11. Jiang B, Monroe SS, Koonin EV, Stine SE, Glass RI. 1993. RNA sequence of astrovirus: distinctive genomic organization and a putative retroviruslike ribosomal frameshifting signal that directs the viral replicase synthesis. Proc. Natl. Acad. Sci. U. S. A. 90:10539-10543.

12. Lewis TL, Greenberg HB, Herrmann JE, Smith LS, Matsui SM. 1994. Analysis of astrovirus serotype 1 RNA, identification of the viral RNAdependent RNA polymerase motif, and expression of a viral structural protein. J. Virol. 68:77-83.

13. Monroe SS, Jiang B, Stine SE, Koopmans M, Glass RI. 1993. Subgenomic RNA sequence of human astrovirus supports classification of Astroviridae as a new family of RNA viruses. J. Virol. 67:3611-3614.

14. Monroe SS, Stine SE, Gorelkin L, Herrmann JE, Blacklow NR, Glass RI. 1991. Temporal synthesis of proteins and RNAs during human astrovirus infection of cultured cells. J. Virol. 65:641-648.

15. Sanchez-Fauquier A, Carrascosa AL, Carrascosa JL, Otero A, Glass RI, Lopez JA, San Martin C, Melero JA. 1994. Characterization of a human astrovirus serotype 2 structural protein (VP26) that contains an epitope involved in virus neutralization. Virology 201:312-320.

16. Willcocks MM, Brown TD, Madeley CR, Carter MJ. 1994. The complete sequence of a human astrovirus. J. Gen. Virol. 75:1785-1788.

17. Bass DM, Upadhyayula U. 1997. Characterization of human serotype 1 astrovirus-neutralizing epitopes. J. Virol. 71:8666-8671.

18. Jonassen CM, Jonassen TO, Saif YM, Snodgrass DR, Ushijima H, Shimizu M, Grinde B. 2001. Comparison of capsid sequences from human and animal astroviruses. J. Gen. Virol. 82:1061-1067.

19. Kakizawa J, Ushijima H, Wen L, Ikeda Y, Oseto M. 1997. Genetic analysis of the capsid region of human astrovirus serotype 3 isolated in Japan. Microbiol. Immunol. 41:637-640.

20. Krishna NK. 2005. Identification of structural domains involved in astrovirus capsid biology. Viral Immunol. 18:17-26.

21. Wang QH, Kakizawa J, Wen LY, Shimizu M, Nishio O, Fang ZY, Ushijima H. 2001. Genetic analysis of the capsid region of astroviruses. J. Med. Virol. 64:245-255.

22. Willcocks MM, Carter MJ. 1993. Identification and sequence determination of the capsid protein gene of human astrovirus serotype 1. FEMS Microbiol. Lett. 114:1-7.

23. Dong J, Dong L, Mendez E, Tao Y. 2011. Crystal structure of the human astrovirus capsid spike. Proc. Natl. Acad. Sci. U. S. A. 108:12681-12686.

24. Mendez E, Fernandez-Luna T, Lopez S, Mendez-Toss M, Arias CF. 2002. Proteolytic processing of a serotype 8 human astrovirus ORF2 polyprotein. J. Virol. 76:7996-8002.

25. Mendez E, Salas-Ocampo E, Arias CF. 2004. Caspases mediate processing of the capsid precursor and cell release of human astroviruses. J. Virol. 78:8601-8608.

26. Dryden KA, Tihova M, Nowotny N, Matsui SM, Mendez E, Yeager M. 2012. Immature and mature human astrovirus: structure, conformational changes, and similarities to hepatitis E virus. J. Mol. Biol. 422:650-658.

27. Guu TS, Liu Z, Ye Q, Mata DA, Li K, Yin C, Zhang J, Tao YJ. 2009. Structure of the hepatitis E virus-like particle suggests mechanisms for virus assembly and receptor binding. Proc. Natl. Acad. Sci. U. S. A. 106: 12992-12997.

28. Li S, Tang X, Seetharaman J, Yang C, Gu Y, Zhang J, Du H, Shih JW, Hew CL, Sivaraman J, Xia N. 2009. Dimerization of hepatitis E virus capsid protein E2s domain is essential for virus-host interaction. PLoS Pathog. 5:e1000537. doi:10.1371/journal.ppat.1000537.

29. Xing L, Li TC, Mayazaki N, Simon MN, Wall JS, Moore M, Wang CY, Takeda N, Wakita T, Miyamura T, Cheng RH. 2010. Structure of hepatitis E virion-sized particle reveals an RNA-dependent viral assembly pathway. J. Biol. Chem. 285:33175-33183.

30. Yamashita T, Mori Y, Miyazaki N, Cheng RH, Yoshimura M, Unno H, Shima R, Moriishi K, Tsukihara T, Li TC, Takeda N, Miyamura T, Matsuura Y. 2009. Biological and immunological characteristics of hepatitis E virus-like particles based on the crystal structure. Proc. Natl. Acad. Sci. U. S. A. 106:12986-12991.
31. Kalia M, Chandra V, Rahman SA, Sehgal D, Jameel S. 2009. Heparan sulfate proteoglycans are required for cellular binding of the hepatitis $\mathrm{E}$ virus ORF2 capsid protein and for viral infection. J. Virol. 83:1271412724

32. Cao S, Lou Z, Tan M, Chen Y, Liu Y, Zhang Z, Zhang XC, Jiang X, Li X, Rao Z. 2007. Structural basis for the recognition of blood group trisaccharides by norovirus. J. Virol. 81:5949-5957.

33. Choi JM, Hutson AM, Estes MK, Prasad BV. 2008. Atomic resolution structural characterization of recognition of histo-blood group antigens by Norwalk virus. Proc. Natl. Acad. Sci. U. S. A. 105:9175-9180.

34. Koci MD, Moser LA, Kelley LA, Larsen D, Brown CC, Schultz-Cherry S. 2003. Astrovirus induces diarrhea in the absence of inflammation and cell death. J. Virol. 77:11798-11808.

35. Otwinowski Z, Minor W. 1997. Processing of X-ray diffraction data collected in oscillation mode. Methods Enzymol. 276:307-326.

36. Adams PD, Afonine PV, Bunkoczi G, Chen VB, Davis IW, Echols N, Headd JJ, Hung LW, Kapral GJ, Grosse-Kunstleve RW, McCoy AJ, Moriarty NW, Oeffner R, Read RJ, Richardson DC, Richardson JS, Terwilliger TC, Zwart PH. 2010. PHENIX: a comprehensive Pythonbased system for macromolecular structure solution. Acta Crystallogr. D Biol. Crystallogr. 66:213-221.

37. Murshudov GN, Vagin AA, Dodson EJ. 1997. Refinement of macromolecular structures by the maximum-likelihood method. Acta Crystallogr. D Biol. Crystallogr. 53:240-255.

38. Emsley P, Cowtan K. 2004. Coot: model-building tools for molecular graphics. Acta Crystallogr. D Biol. Crystallogr. 60:2126-2132.

39. Bonaparte RS, Hair PS, Banthia D, Marshall DM, Cunnion KM, Krishna NK. 2008. Human astrovirus coat protein inhibits serum complement activation via $\mathrm{C} 1$, the first component of the classical pathway. J. Virol. 82:817-827.

40. Caballero S, Guix S, Ribes E, Bosch A, Pinto RM. 2004. Structural requirements of astrovirus virus-like particles assembled in insect cells. J. Virol. 78:13285-13292.

41. Dalton RM, Pastrana EP, Sanchez-Fauquier A. 2003. Vaccinia virus recombinant expressing an 87-kilodalton polyprotein that is sufficient to form astrovirus-like particles. J. Virol. 77:9094-9098.

42. Mendez E, Aguirre-Crespo G, Zavala G, Arias CF. 2007. Association of the astrovirus structural protein VP90 with membranes plays a role in virus morphogenesis. J. Virol. 81:10649-10658.

43. Krissinel E, Henrick K. 2007. Inference of macromolecular assemblies from crystalline state. J. Mol. Biol. 372:774-797.

44. Prasad BV, Hardy ME, Dokland T, Bella J, Rossmann MG, Estes MK. 1999. X-ray crystallographic structure of the Norwalk virus capsid. Science 286:287-290

45. Holm L, Park J. 2000. DaliLite workbench for protein structure comparison. Bioinformatics 16:566-567.

46. Gamblin SJ, Haire LF, Russell RJ, Stevens DJ, Xiao B, Ha Y, Vasisht N, Steinhauer DA, Daniels RS, Elliot A, Wiley DC, Skehel JJ. 2004. The structure and receptor binding properties of the 1918 influenza hemagglutinin. Science 303:1838-1842.

47. Weis W, Brown JH, Cusack S, Paulson JC, Skehel JJ, Wiley DC. 1988. Structure of the influenza virus haemagglutinin complexed with its receptor, sialic acid. Nature 333:426-431.

48. Jindal N, Patnayak DP, Chander Y, Ziegler AF, Goyal SM. 2011. Comparison of capsid gene sequences of turkey astrovirus-2 from poultenteritis-syndrome-affected and apparently healthy turkeys. Arch. Virol. 156:969-977.

49. Herfst S, Schrauwen EJA, Linster M, Chutinimitkul S, de Wit E, Munster VJ, Sorrell EM, Bestebroer TM, Burke DF, Smith DJ, Rimmelzwaan GF, Osterhaus ADME, Fouchier RAM. 2012. Airborne transmission of influenza A/H5N1 virus between ferrets. Science 336:15341541.

50. Imai M, Watanabe T, Hatta M, Das SC, Ozawa M, Shinya K, Zhong G, Hanson A, Katsura H, Watanabe S, Li C, Kawakami E, Yamada S, Kiso M, Suzuki Y, Maher EA, Neumann G, Kawaoka Y. 2012. Experimental adaptation of an influenza H5 HA confers respiratory droplet transmission to a reassortant $\mathrm{H} 5 \mathrm{HA} / \mathrm{H} 1 \mathrm{~N} 1$ virus in ferrets. Nature 486:420-428.

51. Cattoli G, De Battisti C, Toffan A, Salviato A, Lavazza A, Cerioli M, Capua I. 2007. Co-circulation of distinct genetic lineages of astroviruses in turkeys and guinea fowl. Arch. Virol. 152:595-602.

52. De Battisti C, Salviato A, Jonassen CM, Toffan A, Capua I, Cattoli G. 2012. Genetic characterization of astroviruses detected in guinea fowl (Numida meleagris) reveals a distinct genotype and suggests cross-species 
transmission between turkey and guinea fowl. Arch. Virol. 157:13291337.

53. Zhao W, Zhu AL, Yu Y, Yuan CL, Zhu CX, Yang ZB, Cui L, Hua XG. 2011. Complete sequence and genetic characterization of pigeon avian nephritis virus, a member of the family Astroviridae. Arch. Virol. 156: $1559-1565$.
54. Chu DK, Leung CY, Perera HK, Ng EM, Gilbert M, Joyner PH, Grioni A, Ades G, Guan Y, Peiris JS, Poon LL. 2012. A novel group of avian astroviruses in wild aquatic birds. J. Virol. 86:13772-13778.

55. Bidin M, Lojkic I, Bidin Z, Tisljar M, Majnaric D, Mikec M. 2011. Detection and characterization of avian nephritis virus in ducklings. Avian Pathol. 40:173-177. 